\title{
Characterization of Orlicz Sobolev Spaces
}

\section{Tiziano Granucci ${ }^{1}$}

${ }^{1}$ ISIS Leonardo da Vinci, via del Terzolle 91, Firenze, Italy

Correspondence: Tiziano Granucci, ISIS Leonardo da Vinci, via del Terzolle 91, Firenze, Italy.

Received: January 31, 2018 Accepted: February 6, 2018 Online Published: March 13, 2018

doi:10.5539/jmr.v10n2p104 URL: https://doi.org/10.5539/jmr.v10n2p104

\section{Abstract}

We give a characterization of the Orlicz Sobolev spaces $W^{1, \Phi}(\Omega)$ when $\Omega \subset \mathbb{R}^{N}$ is a open subset, $N \geq 1$ and $\Phi \in \triangle^{2}$.

Keywords: Orlicz Spaces, Orlicz Sobolev Spaces, Non-local functionals

\section{Introduction}

In (Gagliardo, 1957) Gagliardo has introduced the semi-norm

$$
[f]_{W^{s, p}(\Omega)}=\left(\int_{\Omega} \int_{\Omega} \frac{|f(x)-f(y)|^{p}}{|x-y|^{N+s p}} d \mathcal{L}^{N}(x) d \mathcal{L}^{N}(y)\right)^{\frac{1}{p}}
$$

besides he has studied the fractional Sobolev spaces $W^{s, p}(\Omega)$ with $0<s<1$ and $p>1$. It is well known that $[f]_{W^{s, p}(\Omega)}$ does not converge to $[f]_{W^{1, p}(\Omega)}=\left(\int_{\Omega}|\nabla f|^{p} d \mathcal{L}^{N}(x)\right)^{\frac{1}{p}}$ when $s \rightarrow 1^{-}$. Moreover, if $\Omega$ is a smooth bounded domain, then in (Bourgain et al.), Bourgain, Brezis and Mironescu have proved that

$$
\lim _{s \rightarrow 1^{-}}(1-s)[f]_{W^{s, p}(\Omega)}^{p}=K_{p, N}[f]_{W^{1, p}(\Omega)}^{p}
$$

for all $f \in W^{1, p}(\Omega)$, with $p>1$. In (Leoni \& Spector, 2011), Leoni and Spector have given an alternative characteization of the Sobolev spaces using the not-local semi-norm

$$
[f]_{W^{1, p}(\Omega)}=\left(\int_{\Omega} \int_{\Omega} \frac{|f(x)-f(y)|^{p}}{|x-y|^{p}} d \mathcal{L}^{N}(x) d \mathcal{L}^{N}(y)\right)^{\frac{1}{p}}
$$

Particularly, in (Leoni \& Spector, 2011), Leoni and Spector have shown the following theorem.

Theorem 1.1. Let $\Omega \subset \mathbb{R}^{N}$ be open, let $f \in L_{\text {loc }}^{p}(\Omega)$. Then $f \in W^{1, p}$ loc $(\Omega)$ if and only if

$$
\lim _{\lambda \rightarrow 0} \limsup _{\varepsilon \rightarrow 0^{+}} \int_{\Omega_{\lambda}} \int_{\Omega_{\lambda}} \frac{|f(x)-f(y)|^{p}}{|x-y|^{p}} \rho_{\varepsilon}(x-y) d \mathcal{L}^{N}(y) d \mathcal{L}^{N}(x)<+\infty
$$

moreover

$$
\begin{aligned}
& \lim _{\lambda \rightarrow 0} \limsup _{\varepsilon \rightarrow 0^{+}} \int_{\Omega_{\lambda}}\left(\int_{\Omega_{\lambda}} \frac{|f(x)-f(y)|^{p}}{|x-y|^{p}} \rho_{\varepsilon}(x-y) d \mathcal{L}^{N}(y)\right) d \mathcal{L}^{N}(x)= \\
& =K_{N, p} \int_{\Omega}|\nabla f(x)|^{p} d \mathcal{L}^{N}(x) .
\end{aligned}
$$

where $\rho_{\varepsilon}$ is a "good" family of mollifiers.

In this article we will extend such results in the case of Orlicz Sobolev Spaces.

The Orlicz spaces have been introduced both as generalization of the spaces $L^{p}$, both for physical motivations see (Adams, 1975; Astarita \& Marrucci, 1974; Diening \& Ruzika, 2007; Gosez, 1974; Lieberman, 1991; Krasnosel'skij \& Rutickii, 1961; Rao \& Ren, 1991). Particularly, from the nineties many results of regularity are gotten for minima of functionals with general growths defined on Orlicz Sobolev spaces, see (Breit et al., 2011; Cianchi \& Fusco, 1999; Dall'Aglio et al., 1998; Diening et al, 2009; Fuchs, 2011; Fusco \& Sbordone, 1990; Granucci, 2017; Klimov, 2000; Talenti, 1990; Young, 1912). The following ipoptesis will be at the base of our results. 
H-1; $\Phi$ is a N-function and $\Phi \in \Delta_{2} o n(0,+\infty)$.

$\mathbf{H - 2} ; \rho_{\varepsilon}$ is a family of mollifiers such that

$$
\begin{gathered}
\rho_{\varepsilon} \geq 0, \quad \int_{\mathbb{R}^{N}} \rho_{\varepsilon} d \mathcal{L}^{N}=1, \\
\lim _{\varepsilon \rightarrow 0^{+}} \int_{|x|>\delta} \rho_{\varepsilon} d \mathcal{L}^{N}=0 \quad \text { for all } \delta>0 .
\end{gathered}
$$

H-3; There exist $\left\{v_{i}\right\}_{i=1, \ldots, N} \subset \mathbb{R}^{N}$ and a $\delta>0$ such that for all $\sigma_{i} \in C_{\delta}\left(v_{i}\right)$ the set $\left\{\sigma_{i}\right\}_{i=1, \ldots, N}$ is linearly independent, where

$$
C_{\delta}(v)=\left\{w \in \mathbb{R}^{N}-\{0\}: \frac{v}{|v|} \cdot \frac{w}{|w|}>1-\delta\right\}
$$

and

$$
\liminf _{\varepsilon \rightarrow 0^{+}} \int_{C_{\delta}\left(v_{i}\right)} \rho_{\varepsilon} d \mathcal{L}^{N}>0
$$

for all $i=1, \ldots, N$.

$\mathbf{H - 4 ;} \rho_{\varepsilon}$ is radial, that is $\rho_{\varepsilon}(x)=\rho_{\varepsilon}(|x|)$ for all $x \in \mathbb{R}^{N}$.

The purpose of our article is to show the followings results.

Theorem 1.2. Let $\Omega \subset \mathbb{R}^{N}$ be open, let $\Phi$ and $\rho_{\varepsilon}$ satisfy $H-1, H-2$ and $H-3$, let $f \in L_{\text {loc }}^{\Phi}(\Omega)$. Then $f \in W^{1} L_{\text {loc }}^{\Phi}(\Omega)$ if and only if

$$
\lim _{\lambda \rightarrow 0} \limsup _{\varepsilon \rightarrow 0^{+}} \int_{\Omega_{\lambda}} \int_{\Omega_{\lambda}} \Phi\left(\frac{|f(x)-f(y)|}{|x-y|}\right) \rho_{\varepsilon}(x-y) d \mathcal{L}^{N}(y) d \mathcal{L}^{N}(x)<+\infty .
$$

Moreover, if $\rho_{\varepsilon}$ satisfy $H-4$ and $\Phi \in \triangle_{2}^{m}$ on $(0,+\infty)$, then there exist $k_{m, N}>0$ such that

$$
\begin{aligned}
& \lim _{\lambda \rightarrow 0^{+}} \lim _{\varepsilon \rightarrow 0^{+}} \int\left(\int_{\Omega_{\lambda}} \Phi\left(\frac{|f(x)-f(y)|}{|x-y|}\right) \rho_{\varepsilon}(x-y) d \mathcal{L}^{N}(y)\right) d \mathcal{L}^{N}(x)= \\
& =k_{m, N} \int_{\Omega} \Phi(|\nabla f(x)|) d \mathcal{L}^{N}(x) .
\end{aligned}
$$

Theorem 1.3. Let $\Omega \subset \mathbb{R}^{N}$ be open, let $\Phi$ and $\rho_{\varepsilon}$ satisfy $H-1, H-2, H-3$ and $H-4$, let $f \in L_{\text {loc }}^{\Phi}(\Omega)$. Assume

$$
\limsup _{\varepsilon \rightarrow 0^{+}} \int_{\Omega} \int_{\Omega} \Phi\left(\frac{|f(x)-f(y)|}{d_{\Omega}(x, y)}\right) \rho_{\varepsilon}\left(d_{\Omega}(x, y)\right) d \mathcal{L}^{N}(y) d \mathcal{L}^{N}(x)<+\infty .
$$

then $f \in W^{1} L_{\text {loc }}^{\Phi}(\Omega)$.

The Theorem 1.2 and the Theorem 1.3 are an alternative characterization of the spaces of Orlicz Sobolev using non local relations, such relations are at the base of numerous results for not local functionals with standard growths and for functionals defined on fractional Sobolev space, see (Bourgain et al., 2001; Di Castro et al., 2016; Di Castro et al., 2014; Maz'ya \& Shaposhnikova, 2002; Mengesha \& Spector; Milman, 2005; Ponce, 2004; Schikarra et al.; Shieh \& Spector). The generalization of these theorems seems to point out the possibility to also extend such results in more general cases.

\section{N-function and Orlicz Spaces}

Definition 2.1. A continuous and convex function $\Phi:[0,+\infty) \rightarrow[0,+\infty)$ is called $N$-function (or Young function) if it satisfies

$$
\begin{aligned}
& \Phi(0)=0 \text { and } \Phi(t)>0 \text { if } t>0 ; \\
& \lim _{\substack{t \rightarrow 0^{+} \\
\Phi(t)}}=0 ; \\
& \lim _{t \rightarrow+\infty} \frac{\Phi(t)}{t}=+\infty .
\end{aligned}
$$


For exemple the function $\Phi_{p, \beta}(t)=t^{p} \ln ^{\beta}(1+t)$, for $p>1$ and $\beta \geq 0$ or $p=1$ and $\beta>0$, is a $\mathrm{N}$-function.

Actually, only the growth at infinity really matters in the definition of $\mathrm{N}$-function.

Indeed, given a continuous and convex function $A:[0,+\infty) \rightarrow[0,+\infty)$ satisfying

$$
\lim _{t \rightarrow+\infty} \frac{A(t)}{t}=+\infty
$$

there exist a $\mathrm{N}$-function $\Phi$ and $t_{0}>0$ such that for every $t>t_{0}$ there holds

$$
A(t)=\Phi(t) .
$$

The function $A$ is called principal part of the $\mathrm{N}$-function $\Phi$. For exemple there exists a $\mathrm{N}$-function $\Phi$ such that $\Phi(t)=t^{\ln (t)}$ near infinity or there exists a $\mathrm{N}$-function $\Phi$ such that $\Phi(t)=t \ln (t)$ near infinity.

The function $A$ is called principal part of the $N$-function $\Phi$. For exemple there exists a $N$-function $\Phi$ such that $\Phi(t)=t^{\ln (t)}$ near infinity or there exists a $N$-function $\Phi$ such that $\Phi(t)=t \ln (t)$ near infinity.

Definition 2.2. If $\Phi_{1}$ and $\Phi_{2}$ are two $N$-functions we say that $\Phi_{1}$ dominates $\Phi_{2}$ near infinity if there exists positive constants $\varkappa$ and $t_{0}$ such that

$$
\Phi_{2}(t) \leq \Phi_{1}(\varkappa t)
$$

for all $t \geq t_{0}$.

Definition 2.3. If $\Phi_{1}$ and $\Phi_{2}$ are two $N$-functions we say that $\Phi_{1}$ and $\Phi_{2}$ are equivalent near infinity $\left(\Phi_{1} \sim \Phi_{2}\right)$ if and only if there exists positive constants $\varkappa_{1}, \varkappa_{2}$ and $t_{0}$ such that

$$
\Phi_{1}\left(\varkappa_{1} t\right) \leq \Phi_{2}(t) \leq \Phi_{1}\left(\varkappa_{2} t\right)
$$

for all $t \geq t_{0}$.

Remark 2.4. If $0<\lim _{t \rightarrow+\infty} \frac{\Phi_{1}(t)}{\Phi_{2}(t)}<+\infty$ then $\Phi_{1}$ and $\Phi_{2}$ are equivalent near infinity.

Let us introduce two important classes of $\mathrm{N}$-functions.

Definition 2.5. A $N$-function $\Phi$ is of class $\Delta_{2}\left(\Phi \in \Delta_{2}\right)$ if exist $k>1$ and $t_{0}>0$ such that

$$
\Phi(2 t) \leq k \Phi(t) \quad \forall t \in\left(t_{0},+\infty\right) .
$$

Definition 2.6. A $N$-function $\Phi$ is of class $\triangle_{2}^{m}\left(\Phi \in \triangle_{2}^{m}\right)$, with $m>1$, if exists $t_{0}>0$ such that for every $\lambda>1$

$$
\Phi(\lambda t) \leq \lambda^{m} \Phi(t) \quad \forall t \in\left(t_{0},+\infty\right) .
$$

Definition 2.7. A $N$-function $\Phi$ is of class $\Delta_{2}$ globally in $(0,+\infty)$ if $(2.2)$ holds for every $t>0$.

Definition 2.8. A $N$-function $\Phi$ is of class $\triangle_{2}^{m}$ globally in $(0,+\infty)$, with $m>1$, if (2.3) holds for every $t>0$.

Remark 2.9. If $\Phi$ is a $N$-function and $\Phi \in \Delta_{2}$ then there exists a $N$-function $\Phi_{1}$ such that $\Phi \sim \Phi_{1}$ and $\Phi_{1} \in \Delta_{2}$ globally in $(0,+\infty)$, see (Krasnosel'skij E Rutickii, 1961; Rao E Ren, 1991).

Definition 2.10. A $N$-function $\Phi$ is of class $\nabla_{2}\left(\Phi \in \nabla_{2}\right)$ if exist $l>1$ and $t_{0}>0$ such that

$$
\Phi(t) \leq \frac{\Phi(l t)}{2 l} \quad \forall t \in\left(t_{0},+\infty\right) .
$$

Definition 2.11. A $N$-function $\Phi$ is of class $\nabla_{2}^{r}\left(\Phi \in \nabla_{2}^{r}\right)$, with $r>1$, if exists $t_{0}>0$ such that for every $\lambda>1$

$$
\lambda^{r} \Phi(t) \leq \Phi(\lambda t) \quad \forall t \in\left(t_{0},+\infty\right) .
$$

Definition 2.12. A $N$-function $\Phi$ is of class $\nabla_{2}$ globally in $(0,+\infty)$ if $(2.4)$ holds for every $t>0$.

Definition 2.13. A $N$-function $\Phi$ is of class $\nabla_{2}^{r}$ globally in $(0,+\infty)$, with $r>1$, if (2.5) holds for every $t>0$.

Remark 2.14. If $\Phi$ is a $N$-function and $\Phi \in \nabla_{2}$ then there exists a $N$-function $\Phi_{1}$ such that $\Phi \sim \Phi_{1}$ and $\Phi_{1} \in \nabla_{2}$ globally in $(0,+\infty)$, see (Krasnosel'skij $\mathcal{E}$ Rutickii, 1961; Rao $\mathcal{E}$ Ren, 1991). 
The $N$-functions $\Phi \in \triangle_{2}^{m}$ are characterized by the following result.

Lemma 2.15. Let $\Phi$ be a $N$-function and let $\dot{\Phi}_{+}$be its right derivative. For $m>1$ the following properties are equivalent:

(i) $\Phi(\lambda t) \leq \lambda^{m} \Phi(t)$, for every $t>00$, for every $\lambda>1$;

(ii) $t \dot{\Phi}_{+}(t) \leq m \Phi(t)$, for every $t>0$;

(iii) the function $\frac{\Phi(t)}{t^{m}}$ is nonincreasing on $(0,+\infty)$.

Proof. See (Dall'Aglio et al., 1998; Krasnosel'skij \& Rutickii, 1961; Rao \& Ren, 1991).

The $N$-functions $\Phi \in \nabla_{2}^{r}$ are characterized by the following result.

Lemma 2.16. Let $\Phi$ be a $N$-function and let $\dot{\Phi}_{-}$be its left derivative. For $r>1$ the following properties are equivalent:

(i)' $\Phi(\lambda t) \geq \lambda^{r} \Phi(t)$, for every $t>0$, for every $\lambda>1$;

(ii)' $t \dot{\Phi}_{-}(t) \geq r \Phi(t)$, for every $t>0$;

(iii)' the function $\frac{\Phi(t)}{t^{r}}$ is nondecreasing on $(0,+\infty)$.

Proof. See (Dall'Aglio et al., 1998; Krasnosel'skij \& Rutickii, 1961; Rao \& Ren, 1991).

Remark 2.17. We observe that

$$
\Delta_{2}=\bigcup_{m>1} \triangle_{2}^{m}
$$

and

$$
\nabla_{2}=\bigcup_{r>1} \nabla_{2}^{r} .
$$

Remark 2.18. If $\Phi$ is a $N$-function then $t \dot{\Phi}_{-}(t) \geq r \Phi(t)$ for every $t>0$. By Lemma 2.16 it follows that $\Phi \in \nabla_{2}^{1}$ on $(0,+\infty)$.

Now we give some alternative characterizations of the N-funtions of class $\triangle_{2}^{m}$ globally in $(0,+\infty)$ and of class $\nabla_{2}^{r}$ globally in $(0,+\infty)$.

Proposition 2.19. $\Phi$ is a $N$-function of class $\triangle_{2}^{m}$ globally in $(0,+\infty)$ if and only if $\Phi^{-1}(a w) \leq a^{\frac{1}{m}} \Phi^{-1}(w)$ for every $w \in(0,+\infty)$ and $a \in(0,1)$.

Proof. If $\Phi$ is a N-function of class $\triangle_{2}^{m}$ globally in $(0,+\infty)$, then we have $\Phi(\lambda t) \leq \lambda^{m} \Phi(t)$ for every $t \in(0,+\infty)$ and $\lambda>1$. Let us put $t=\frac{s}{\lambda}$ then we have $\frac{\Phi(s)}{\lambda^{m}} \leq \Phi\left(\frac{s}{\lambda}\right)$ and $\Phi^{-1}\left(\frac{\Phi(s)}{\lambda^{m}}\right) \leq \frac{s}{\lambda}$ for every $s \in(0,+\infty)$ and $\lambda>1$. Let us put $s=\Phi^{-1}(w)$ then we have $\Phi^{-1}\left(\frac{w}{\lambda^{m}}\right) \leq \frac{\Phi^{-1}(w)}{\lambda}$ for every $w \in(0,+\infty)$ and $\lambda>1$. Let us put $\frac{1}{\lambda^{m}}=a$ then we have $\Phi^{-1}(a w) \leq a^{\frac{1}{m}} \Phi^{-1}(w)$ for every $w \in(0,+\infty)$ and $a \in(0,1)$. The converse follows in a similar manner.

Proposition 2.20. $\Phi$ is a $N$-function of class $\Delta_{2}^{m}$ globally in $(0,+\infty)$ if and only if $\lambda \Phi^{-1}(s) \leq \Phi^{-1}\left(\lambda^{m} s\right)$ for every $s \in$ $(0,+\infty)$ and $\lambda>1$.

Proof. If $\Phi$ is a $\mathrm{N}$-function of class $\Delta_{2}^{m}$ globally in $(0,+\infty)$, then $\Phi(\lambda t) \leq \lambda^{m} \Phi(t)$ for every $t \in(0,+\infty)$ and $\lambda>1$. It follows that $\lambda t \leq \Phi^{-1}\left(\lambda^{m} \Phi(t)\right)$ then if $t=\Phi^{-1}(s)$ we get $\lambda \Phi^{-1}(s) \leq \Phi^{-1}\left(\lambda^{m} s\right)$ for every $s \in(0,+\infty)$ and $\lambda>1$. The converse follows in a similar manner.

Now we get the following characterization of the N-functions of class $\triangle_{2}^{m}$ globally in $(0,+\infty)$, this characterization is not present in the bibliography note to the author and we will use it in the paper.

Proposition 2.21. $\Phi$ is a $N$-function of class $\triangle_{2}^{m}$ globally in $(0,+\infty)$ if and only if $a^{m} \Phi(s) \leq \Phi($ as $)$ for every $s \in(0,+\infty)$ and $a \in(0,1)$.

Proof. If $\Phi$ is a N-function of class $\triangle_{2}^{m}$ globally in $(0,+\infty)$, then $\Phi(\lambda t) \leq \lambda^{m} \Phi(t)$ for every $t \in(0,+\infty)$ and $\lambda>1$. It follows that $\frac{1}{\lambda^{m}} \Phi(\lambda t) \leq \Phi(t)$ then if $t=\frac{s}{\lambda}$ we get $\frac{1}{\lambda^{m}} \Phi(s) \leq \Phi\left(\frac{s}{\lambda}\right)$ for every $s \in(0,+\infty)$ and $\lambda>1$. If we put $a=\frac{1}{\lambda} \in(0,1)$ we get $a^{m} \Phi(s) \leq \Phi(a s)$ for every $s \in(0,+\infty)$ and $a \in(0,1)$. The converse follows in a similar manner. 
Now we get the following characterization of the $\mathrm{N}$-functions of class $\nabla_{2}^{r}$ globally in $(0,+\infty)$, this characterization is not present in the bibliography note to the author.

Proposition 2.22. $\Phi$ is a $N$-function of class $\nabla_{2}^{r}$ globally in $(0,+\infty)$ if and only if

$$
\Phi^{-1}\left(\frac{w}{\lambda^{r}}\right) \geq \frac{\Phi^{-1}(w)}{\lambda}
$$

for every $w \in(0,+\infty)$ and $\lambda>1$.

Proof. If $\Phi$ is a N-function of class $\nabla_{2}^{r}$ globally in $(0,+\infty)$, then we have $\lambda^{r} \Phi(t) \leq \Phi(\lambda t)$ for every $t \in(0,+\infty)$ and $\lambda>1$. Let us put $t=\frac{s}{\lambda}$ then we have $\frac{\Phi(s)}{\lambda^{r}} \geq \Phi\left(\frac{s}{\lambda}\right)$ and $\Phi^{-1}\left(\frac{\Phi(s)}{\lambda^{r}}\right) \geq \frac{s}{\lambda}$ for every $s \in(0,+\infty)$ and $\lambda>1$. Let us put $s=\Phi^{-1}(w)$ then we have $\Phi^{-1}\left(\frac{w}{\lambda^{r}}\right) \geq \frac{\Phi^{-1}(w)}{\lambda}$ for every $w \in(0,+\infty)$ and $\lambda>1$. The converse follows in a similar manner.

Remark 2.23. If we choose $\lambda=\kappa^{\frac{1}{r}}>1$ then we can write the inequality (2.6) this way

$$
\Phi^{-1}\left(\frac{w}{\kappa}\right) \geq \frac{\Phi^{-1}(w)}{\kappa^{\frac{1}{r}}}
$$

for every $w \in(0,+\infty)$ and $\kappa>1$.

Another characterization of the functions $\mathrm{N}$-function of class $\nabla_{2}^{r}$ globally in $(0,+\infty)$, it is the following.

Proposition 2.24. $\Phi$ is a $N$-function of class $\nabla_{2}^{r}$ globally in $(0,+\infty)$ if and only if $\Phi(a t) \leq a^{r} \Phi(t)$ for every $t \in(0,+\infty)$ and $a \in(0,1)$.

Proof. If $\Phi$ is a $\mathrm{N}$-function of class $\nabla_{2}^{r}$ globally in $(0,+\infty)$, then we have $\lambda^{r} \Phi(t) \leq \Phi(\lambda t)$ for every $t \in(0,+\infty)$ and $\lambda>1$. If we put $t=\frac{s}{\lambda}$ we get $\Phi\left(\frac{s}{\lambda}\right) \leq \frac{1}{\lambda^{r}} \Phi(s)$, then if $a=\frac{1}{\lambda}$ it follows that $\Phi(a s) \leq a^{r} \Phi(s)$ for every $s \in(0,+\infty)$ and $a \in(0,1)$. The converse follows in a similar manner.

Proposition 2.25. $\Phi$ is a $N$-function of class $\nabla_{2}^{r}$ globally in $(0,+\infty)$ if and only if $\Phi^{-1}(a w) \geq a^{\frac{1}{r}} \Phi^{-1}(w)$ for every $w \in(0,+\infty)$ and $a \in(0,1)$.

Proof. If $\Phi$ is a $\mathrm{N}$-function of class $\nabla_{2}^{r}$ globally in $(0,+\infty)$, then we have $\lambda^{r} \Phi(t) \leq \Phi(\lambda t)$ for every $t \in(0,+\infty)$ and $\lambda>1$. Let us put $t=\frac{s}{\lambda}$ then we have $\frac{\Phi(s)}{\lambda^{r}} \geq \Phi\left(\frac{s}{\lambda}\right)$ and $\Phi^{-1}\left(\frac{\Phi(s)}{\lambda^{r}}\right) \geq \frac{s}{\lambda}$ for every $s \in(0,+\infty)$ and $\lambda>1$. Let us put $s=\Phi^{-1}(w)$ then we have $\Phi^{-1}\left(\frac{w}{\lambda^{r}}\right) \geq \frac{\Phi^{-1}(w)}{\lambda}$ for every $w \in(0,+\infty)$ and $\lambda>1$. Let us put $\frac{1}{\lambda^{r}}=a$ then we have $\Phi^{-1}(a w) \geq a^{\frac{1}{r}} \Phi^{-1}(w)$ for every $w \in(0,+\infty)$ and $a \in(0,1)$. The converse follows in a similar manner.

Proposition 2.26. $\Phi$ is a $N$-function of class $\nabla_{2}^{r}$ globally in $(0,+\infty)$ if and only if $\Phi^{-1}\left(\lambda^{r} s\right) \leq \lambda \Phi^{-1}(s)$ for every $s \in$ $(0,+\infty)$ and $\lambda>1$.

Proof. f $\Phi$ is a N-function of class $\nabla_{2}^{r}$ globally in $(0,+\infty)$, then we have $\lambda^{r} \Phi(t) \leq \Phi(\lambda t)$ for every $t \in(0,+\infty)$ and $\lambda>1$. It follows that $\Phi^{-1}\left(\lambda^{r} \Phi(t)\right) \leq \lambda t$ then if $t=\Phi^{-1}(s)$ we get $\Phi^{-1}\left(\lambda^{r} s\right) \leq \lambda \Phi^{-1}(s)$ for every $s \in(0,+\infty)$ and $\lambda>1$. The converse follows in a similar manner.

Remark 2.27. $f \Phi$ is a $N$-function and $\Phi \in \Delta_{2}$ then there exists a $N$-function $\Phi_{1}$ such that $\Phi \sim \Phi_{1}$ and $\Phi_{1}$, is a $N$-function of class $\triangle_{2}^{m}$ globally in $(0,+\infty)$.

Lemma 2.28. Let $g(t), h(t)$ be a non-negative and increasing functions on $[0,+\infty)$ then

$$
g(t) h(s) \leq g(t) h(t)+g(s) h(s)
$$

for every $s, t \in[0,+\infty)$.

Proof. If $s \leq t$ then $g(t) h(s) \leq g(t) h(t) \leq g(t) h(t)+g(s) h(s)$. If $t \leq s$ then $g(t) h(s) \leq g(s) h(s) \leq g(t) h(t)+$ $g(s) h(s)$. 
Remark 2.29. Since $\dot{\Phi}(t) \leq \dot{\Phi}_{+}(t)$ for every $t>0$, where $\dot{\Phi}(t)$ is the weak derivative of $\Phi$ and $\dot{\Phi}_{+}(t)$ is the right derivative of $\Phi$, then by Lemma 2 and Lemma 4 we have

$$
a \dot{\Phi}(b) \leq a \dot{\Phi}_{+}(b) \leq a \dot{\Phi}_{+}(a)+b \dot{\Phi}_{+}(b) \leq m(\Phi(a)+\Phi(b)) .
$$

Now we can introduce Orlicz spaces and Orlicz Sobolev Spaces, $L^{\Phi}$ and $W^{1} L^{\Phi}$. Let $\Omega \subseteq \mathbb{R}^{N}$ be a bounded and open set, the Orlicz class $K^{\Phi}(\Omega)$ is the set of all measurable functions $u: \Omega \rightarrow \mathbb{R}$ (equivalence classes modulo equality $\mathcal{L}^{N}$ a.e. in $\Omega$ ) satisfying $\int_{\Omega} \Phi(|u|) d \mathcal{L}^{N}<+\infty$. The Orlicz space $L^{\Phi}(\Omega)$ is defined to be the linear hull of $K^{\Phi}(\Omega)$, thus it consists of all measurable functions $u$ such that $\lambda u \in K^{\Phi}(\Omega)$ for some $\lambda>0$. Moreover, the equality $K^{\Phi}(\Omega) \equiv L^{\Phi}(\Omega)$ holds if and only if $\Phi \in \triangle_{2}$.

Definition 2.30. If $\Omega \subset \mathbb{R}^{N}$ is a bounded open set and $\Phi \in \Delta_{2}$ then we define

$$
W^{1} L^{\Phi}(\Omega)=\left\{u \in L^{\Phi}(\Omega): \partial_{i} u \in L^{\Phi}(\Omega) \text { for } i=1, \ldots, N\right\}
$$

where $\partial_{i} u$ are the weak derivatives of $u$ for $i=1, \ldots, N$.

Theorem 2.31. Let $\Phi \in \triangle_{2}$, then $L^{\Phi}(\Omega)$ and $W^{1} L^{\Phi}(\Omega)$ are Banach spaces with the following norms

$$
\|u\|_{\Phi, \Omega}=\inf \left(k>0: \int_{\Omega} \Phi\left(\frac{|u|}{k}\right) d \mathcal{L}^{N} \leq 1\right)
$$

and

$$
\|u\|_{1, \Phi, \Omega}=\|u\|_{\Phi, \Omega}+\sum_{i=1}^{N}\left\|\partial_{i} u\right\|_{\Phi, \Omega}
$$

We observe that if $\Phi(t)=t^{p}$, with $p>1$, then $\|u\|_{\Phi, \Omega}=\|u\|_{p, \Omega}$, where $\|u\|_{p, \Omega}=\int_{\Omega}|u|^{p} d \mathcal{L}^{N}$. In general, however, so simple relationships do not be had among the Luxemburg norm $\|u\|_{\Phi, \Omega}$ and the integral $\int_{\Omega} \Phi(|u|) d \mathcal{L}^{N}$, this creates some difficulties to use the Luxemburg norm and the Hölder inequality then we are forced to introduce some suitable tricks to proceed. For greater details on Orlicz spaces, Orlicz-Sobolev spaces and Luxemburg norm we refer (Adams, 1975; Krasnosel'skij \& Rutickii, 1961; Rao \& Ren, 1991)

\section{Lemmas}

Fix $\psi \in C_{c}^{\infty}\left(\mathbb{R}^{N}\right)$ such that $\int_{\mathbb{R}^{N}} \psi d x=1$ and $\operatorname{supp}(\psi) \Subset B_{1}(0)$. For $\delta>0$ define

$$
\psi_{\delta}(x)=\frac{1}{\delta^{N}} \psi\left(\frac{x}{\delta}\right)
$$

Given a open set $\Omega \subset \mathbb{R}^{N}$ and a function $f \in L_{l o c}^{1}(\Omega)$, for every $x \in \Omega_{\delta}=\{x \in \Omega: \operatorname{dist}(x, \partial \Omega)>\delta\}$ define the mollification of the function $f$ by

$$
f_{\delta}(x)=\left(f * \psi_{\delta}\right)(x)=\int_{\mathbb{R}^{N}} f(y) \psi_{\delta}(x-y) d y
$$

Remark 3.1. Since $\Phi$ is a convex function then

$$
\Phi(u) \geq \Phi(a)+\dot{\Phi}(a)(u-a)
$$

for every $u, a>0$. Since $\psi_{\delta}(x-y) \geq 0$ we get

$$
\psi_{\delta}(x-y) \Phi(u) \geq \psi_{\delta}(x-y) \Phi(a)+\psi_{\delta}(x-y) \dot{\Phi}(a)(u-a)
$$

If we choose $u=|f(y)|$ and $a=\int_{\mathbb{R}^{N}}|f(z)| \psi_{\delta}(x-z) d z$ and if we integrate on $\mathbb{R}^{N}$, since $\int_{\mathbb{R}^{N}} \psi_{\delta}(z-y) d z=1$, it follows

$$
\int_{\mathbb{R}^{N}} \Phi\left(|f(y)| \psi_{\delta}(x-y)\right) d y \geq \Phi\left(\int_{\mathbb{R}^{N}}|f(y)| \psi_{\delta}(x-y) d y\right)
$$

for every $x \in \Omega$ with dist $(x, \partial \Omega)>\delta$. 
Lemma 3.2. Let $\rho_{\varepsilon}$ satisfy $H-2$ and $H-3$, let $\left\{\mu_{\varepsilon}\right\} \subset \mathcal{M}\left(S^{N-1}\right)$ the measure defined by

$$
\mu_{\varepsilon}(F)=\int_{F} \int_{0}^{+\infty} \rho_{\varepsilon}(t \sigma) t^{N-1} d t d \mathcal{H}^{N-1}(\sigma)
$$

for every Borel subset $F \subset S^{N-1}$. Then there exist a subsequence $\varepsilon_{j}$, with $\varepsilon_{j} \rightarrow 0^{+}, \mu \in \mathcal{M}\left(S^{N-1}\right)$ such that $\mu_{\varepsilon_{j}} \stackrel{*}{\rightarrow} \mu$ in $\mathcal{M}\left(S^{N-1}\right)$ for $\varepsilon_{j} \rightarrow 0^{+}$. Moreover, for every $N$-function $\Phi \in \Delta_{2}$, there exists $\alpha_{\Phi}>0$ such that for every $v \in \mathbb{R}^{N}$ we have

$$
\int_{S^{N-1}} \Phi(|v \cdot \sigma|) d \mu(\sigma) \geq \alpha_{\Phi} \Phi(|v|) .
$$

Proof. Using polar coordinates and $\mathrm{H}-2$ we have

$$
\mu_{\varepsilon}\left(S^{N-1}\right)=\int_{S^{N-1}} \int_{0}^{+\infty} \rho_{\varepsilon}(t \sigma) t^{N-1} d t d \mathcal{H}^{N-1}(\sigma)=\int_{\mathbb{R}^{N}} \rho_{\varepsilon} d \mathcal{L}^{N}=1
$$

then $\left\|\mu_{\varepsilon}\right\|_{\mathcal{M}\left(S^{N-1}\right)}=1$ and so there exists $\mu \in \mathcal{M}\left(S^{N-1}\right)$ such that $\mu_{\varepsilon} \stackrel{*}{\rightarrow} \mu$ in $\mathcal{M}\left(S^{N-1}\right)$ and $\|\mu\|_{\mathcal{M}\left(S^{N-1}\right)}=1$. Let $\{v\}_{i=1, \ldots, N}$ be the linearly independent set of vectors given in $\mathrm{H}-3$, We claim there exists $\varepsilon_{0}>0$ with the property that for all $v \in \mathbb{R}^{N}$ there exists an $i$ such that

$$
|v \cdot \sigma| \geq \varepsilon_{0}|v|
$$

for all $\sigma \in C_{\delta}\left(v_{i}\right) \cap S^{N-1}$. By rescaling we restrict ourselves to the case $v \in S^{N-1}$, and we proceed by contradiction. If not, then there exist a sequence $\left\{\varepsilon_{k}\right\}_{k \in \mathbb{N}}$ tending to zero, $w_{k} \in S^{N-1}$ and $\sigma_{i, k} \in C_{\delta}\left(v_{i}\right), i=1, \ldots, N$, so that up to a subsequence, which we will not relabel, $w_{k} \rightarrow w_{0} \in S^{N-1}$ and $\sigma_{i, k} \rightarrow \sigma_{i, 0} \in C_{\delta}\left(v_{i}\right)$, with $\left|w_{0} \cdot \sigma_{i, 0}\right|=0$ for all $i=1, \ldots, N$. However, since $\left\{\sigma_{i, 0}\right\}_{i=1, \ldots, N}$ form a linearly independet set, (see Remark 1.4 of (Leoni \& Spector, 2011)), we have a contradiction. Definne

$$
c=\min _{i=1, \ldots, N} \liminf _{j \rightarrow+\infty} \int_{C_{\delta}\left(v_{i}\right)} \rho_{\varepsilon_{j}} d \mathcal{L}^{N}
$$

then $c>0$. Given $v \in \mathbb{R}^{N}$, let $i$ such that $|v \cdot \sigma| \geq \varepsilon_{0}|v|$ for all $\sigma \in C_{\delta}\left(v_{i}\right) \cap S^{N-1}$, then

$$
\begin{aligned}
\int_{S^{N-1}} \Phi(|v \cdot \sigma|) d \mu_{\varepsilon_{j}}(\sigma) & \geq \int_{C_{\delta}\left(v_{i}\right) \cap S^{N-1}} \Phi(|v \cdot \sigma|) d \mu_{\varepsilon_{j}}(\sigma) \\
& \geq \int_{C_{\delta}\left(v_{i}\right) \cap S^{N-1}} \Phi\left(\varepsilon_{0}|v|\right) d \mu_{\varepsilon_{j}}(\sigma) \\
& =\Phi\left(\varepsilon_{0}|v|\right) \mu_{\varepsilon_{j}}\left(C_{\delta}\left(v_{i}\right) \cap S^{N-1}\right) \\
& =\Phi\left(\varepsilon_{0}|v|\right) \int_{C_{\delta}\left(v_{i}\right) \cap \cap S^{N-1}} \int_{0}^{+\infty} \rho_{\varepsilon}(t \sigma) t^{N-1} d t d \mathcal{H}^{N-1}(\sigma)
\end{aligned}
$$

By Tonelli's theorem we get

$$
\int_{S^{N-1}} \Phi(|v \cdot \sigma|) d \mu_{\varepsilon_{j}} \geq \Phi\left(\varepsilon_{0}|v|\right) \int_{C_{\delta}\left(v_{i}\right)} \rho_{\varepsilon_{j}} d \mathcal{L}^{N} \geq c \Phi\left(\varepsilon_{0}|v|\right)
$$

Letting $j \rightarrow+\infty$, since $\mu_{\varepsilon} \stackrel{*}{\rightarrow} \mu$ in $\mathcal{M}\left(S^{N-1}\right)$, it follows

$$
\int_{S^{N-1}} \Phi(|v \cdot \sigma|) d \mu(\sigma) \geq c \Phi\left(\varepsilon_{0}|v|\right)
$$

since $\Phi$ is a N function, then by Remark refR $\Phi \in \nabla_{2}^{1}$ and using Lemma 2.16 (i) and Proposition 2.21 we have

$$
\int_{S^{N-1}} \Phi(|v \cdot \sigma|) d \mu(\sigma) \geq c\left(\varepsilon_{0}\right) \Phi(|v|)
$$

where $c\left(\varepsilon_{0}\right)=c \min \left\{\varepsilon_{0}, \varepsilon_{0}^{m}\right\}$ 
Definition 3.3. For every fixed $\eta>0$ we define

$$
\rho_{\varepsilon}^{\eta}=\rho_{\varepsilon} \chi_{B_{\eta}(0)}
$$

We hav the following properties of $\rho_{\varepsilon}^{\eta}$

$$
\begin{gathered}
\rho_{\varepsilon}^{\eta}<\rho_{\varepsilon}, \\
\rho_{\varepsilon}^{\eta} \geq 0, \quad \int_{\mathbb{R}^{N}} \rho_{\varepsilon}^{\eta} d \mathcal{L}^{N} \leq 1, \\
\lim _{\varepsilon \rightarrow 0^{+}} \int_{|x|>\delta} \rho_{\varepsilon}^{\eta} d \mathcal{L}^{N}=0 \quad \text { for all } \delta>0, \\
\lim _{\varepsilon \rightarrow 0^{+}} \int_{E}|x| \rho_{\varepsilon}^{\eta}(x) d \mathcal{L}^{N}(x)=0
\end{gathered}
$$

for every $E \subset \mathbb{R}^{N}$ bounded and measurable. Now we can define the measure

$$
\mu_{\varepsilon, \eta}(F)=\int_{F} \int_{0}^{+\infty} \rho_{\varepsilon}^{\eta}(t \sigma) t^{N-1} d t d \mathcal{H}^{N-1}(\sigma)
$$

for every Borel subset $F \subset S^{N-1}$. Applying the Randon Nikodym theorem, for $\mathcal{H}^{N-1}$ a.e. $\sigma \in S^{N-1}$

$$
\frac{d \mu_{\varepsilon, \eta}}{d \mathcal{H}^{N-1}}(\sigma)=\int_{0}^{+\infty} \rho_{\varepsilon}^{\eta}(t \sigma) t^{N-1} d t=\int_{0}^{\eta} \rho_{\varepsilon}(t \sigma) t^{N-1} d t .
$$

Lemma 3.4. Let $\rho_{\varepsilon}$ satisfy $H-2$, Let $\left\{\mu_{\varepsilon}\right\} \subset \mathcal{M}\left(S^{N-1}\right)$ the measure defined in (3.1). If $\mu_{\varepsilon_{j}} \stackrel{*}{\rightarrow} \mu \in \mathcal{M}\left(S^{N-1}\right)$ for $\varepsilon_{j} \rightarrow 0^{+}$ then, for every $\eta>0, \mu_{\varepsilon, \eta} \stackrel{*}{\rightarrow} \mu \in \mathcal{M}\left(S^{N-1}\right)$, where $\mu_{\varepsilon, \eta}$ are the measures defined in (3.8).

Proof. We begin by proving that $\mu_{\varepsilon_{j}, \eta}-\mu_{\varepsilon_{j}} \rightarrow 0$ in $\mathcal{M}\left(S^{N-1}\right)$. For $f \in C\left(S^{N-1}\right)$, with $\|f\|_{\infty}=1$ we have

$$
\begin{aligned}
\left|\int_{S^{N-1}} f d \mu_{\varepsilon_{j}, \eta}-\int_{S^{N-1}} f d \mu_{\varepsilon_{j}}\right| & =\left|\int_{S^{N-1}} \int_{\eta}^{+\infty} f(\sigma) \rho_{\varepsilon}^{\eta}(t \sigma) t^{N-1} d t d \mathcal{H}^{N-1}(\sigma)\right| \\
& \leq \int_{S^{N-1}} \int_{\eta}^{+\infty} \rho_{\varepsilon_{j}}(t \sigma) t^{N-1} d t d \mathcal{H}^{N-1}(\sigma) \\
& =\int_{|x|>\eta} \rho_{\varepsilon_{j}}(x) d \mathcal{L}^{N}
\end{aligned}
$$

then

$$
\left\|\mu_{\varepsilon_{j}, \eta}-\mu_{\varepsilon_{j}}\right\|_{\mathcal{M}\left(S^{N-1}\right)} \leq \int_{|x|>\eta} \rho_{\varepsilon_{j}}(x) d \mathcal{L}^{N} \rightarrow 0
$$

for $j \rightarrow+\infty$, thus $\mu_{\varepsilon_{j}, \eta}-\mu_{\varepsilon_{j}} \rightarrow 0$ in $\mathcal{M}\left(S^{N-1}\right)$. Since $\mu_{\varepsilon_{j}} \stackrel{*}{\rightarrow} \mu$ in $\mathcal{M}\left(S^{N-1}\right)$, it follows that $\mu_{\varepsilon_{j}, \eta} \stackrel{*}{\rightarrow} \mu$ in $\mathcal{M}\left(S^{N-1}\right)$.

Remark 3.5. By the definition (3.3) of $\rho_{\varepsilon}^{\eta}$ we hace the following properties

$$
\begin{array}{ll}
\rho_{\varepsilon}^{\eta} \geq 0 & \\
\int_{\mathbb{R}^{N}} \rho_{\varepsilon}^{\eta}(x) d \mathcal{L}^{N} \leq 1 & \text { for all } \delta>0 \\
\lim _{\varepsilon \rightarrow 0^{+}} \int_{|x|>\delta} \rho_{\varepsilon}^{\eta}(x) d \mathcal{L}^{N}=0 & \\
\lim _{\varepsilon \rightarrow 0^{+}} \int_{E}|x| \rho_{\varepsilon}^{\eta}(x) d \mathcal{L}^{N}=0 & \text { for every } E \subset \mathbb{R}^{N} \text { bounded and measurable. }
\end{array}
$$

Definition 3.6. We define

$$
E^{r}=\left\{x \in \mathbb{R}^{N}: \operatorname{dist}(x, E)<r\right\}
$$

and

$$
E_{r}=\left\{x \in \mathbb{R}^{N}:|x|<\frac{1}{r} ; \operatorname{dist}(x, \partial E)>r\right\}
$$


Lemma 3.7. Let $A \subset \mathbb{R}^{N}$ be open and bounded and let $f \in C^{2}\left(\overline{A^{\eta}}\right)$ for some $\eta>0$, then

$$
|f(x)-f(y)-\nabla f(x) \cdot(x-y)| \leq C_{f}|x-y|^{2}
$$

for all $x \in A$ and $y \in A^{\eta}$ where $C_{f}>0$ depends upon $\|f\|_{C^{2}\left(\overline{A^{\eta}}\right)}$.

Proof. See (Leoni \& Spector, 2011).

Lemma 3.8. Let $\Omega \subset \mathbb{R}^{N}$ be open, let $\Phi$ and $\rho_{\varepsilon}$ satisfy $H-1, H-2, H-3$, let $A \subset \Omega$ be open and bounded with dist $(A, \partial \Omega)>$ 0 , let $f \in C^{2}\left(\overrightarrow{A^{\eta}}\right)$ where $0<\eta<\operatorname{dist}(A, \partial \Omega)$, let $\varepsilon_{j} \rightarrow 0^{+}$and assume that $\mu_{\varepsilon_{j}} \stackrel{*}{\rightarrow} \mu$ in $\mathcal{M}\left(S^{N-1}\right)$; then for every $x \in A$ we have

$$
\lim _{j \rightarrow+\infty} \int_{A^{\eta}} \Phi\left(\frac{|f(x)-f(y)|}{|x-y|}\right) \rho_{\varepsilon_{j}}^{\eta}(x-y) d \mathcal{L}^{N}(y)=\int_{S^{N-1}} \Phi(|\nabla f(x) \cdot \sigma|) d \mu(\sigma)
$$

where $\rho_{\varepsilon_{j}}^{\eta}$ is the family introduced in (3.3).

Proof. Set $M_{f}=\|\nabla f\|_{L^{\infty}\left(\bar{A}^{\eta}\right)}$, let $0 \leq s, t \leq M_{f}$ then $|\Phi(s)-\Phi(t)| \leq \dot{\Phi}(\xi)|s-t|$, choose $s=\left|\frac{f(x)-f(y)}{|x-y|}\right|$ and $t=\left|\nabla f(x) \frac{x-y}{|x-y|}\right|$ then

$$
\begin{aligned}
\left|\Phi\left(\left|\frac{f(x)-f(y)}{x-y}\right|\right)-\Phi\left(\mid \nabla f(x) \frac{x-y}{|x-y|}\right)\right| & \leq \dot{\Phi}\left(M_{f}\right)|| \frac{f(x)-f(y)}{|x-y|}|-| \nabla f(x) \frac{x-y}{|x-y|}|| \\
& \leq \dot{\Phi}\left(M_{f}\right)\left|\frac{f(x)-f(y)-\nabla f(x)(x-y)}{|x-y|}\right|
\end{aligned}
$$

Using Lemma 3.7 it follows

$$
\left|\Phi\left(\left|\frac{f(x)-f(y)}{x-y}\right|\right)-\Phi\left(\left|\nabla f(x) \frac{x-y}{|x-y|}\right|\right)\right| \leq \dot{\Phi}\left(M_{f}\right) C_{f}|x-y|
$$

therefore

$$
\begin{aligned}
& \int_{A^{\eta}}\left|\Phi\left(\left|\frac{f(x)-f(y)}{x-y}\right|\right)-\Phi\left(\left|\nabla f(x) \frac{x-y}{|x-y|}\right|\right)\right| \rho_{\varepsilon_{j}}^{\eta}(x-y) d \mathcal{L}^{N}(y) \leq \\
& \leq \Phi\left(M_{f}\right) C_{f} \int_{A^{\eta}}|x-y| \rho_{\varepsilon_{j}}^{\eta}(x-y) d \mathcal{L}^{N}(y)
\end{aligned}
$$

By Remark 3.5 we get

$$
\begin{aligned}
& \limsup _{j \rightarrow+\infty} \int_{A^{\eta}} \Phi\left(\left|\frac{f(x)-f(y)}{x-y}\right|\right) \rho_{\varepsilon_{j}}^{\eta}(x-y) d \mathcal{L}^{N}(y)= \\
& =\limsup _{j \rightarrow+\infty} \int_{A^{\eta}} \Phi\left(\left|\nabla f(x) \frac{x-y}{|x-y|}\right|\right) \rho_{\varepsilon_{j}}^{\eta}(x-y) d \mathcal{L}^{N}(y)
\end{aligned}
$$

Since $\rho_{\varepsilon_{j}}^{\eta}(x-y)=0$ if $|x-y|>\eta$ then

$$
\begin{aligned}
\int_{A^{\eta}} \Phi\left(\left|\nabla f(x) \frac{x-y}{|x-y|}\right|\right) \rho_{\varepsilon_{j}}^{\eta}(x-y) d \mathcal{L}^{N}(y) & =\int_{B_{\eta}(x)} \Phi\left(\left|\nabla f(x) \frac{x-y}{|x-y|}\right|\right) \rho_{\varepsilon_{j}}^{\eta}(x-y) d \mathcal{L}^{N}(y) \\
& =\int_{S^{N-1}} \Phi(|\nabla f(x) \cdot \sigma|) \int_{\eta}^{+\infty} \rho_{\varepsilon}^{\eta}(t \sigma) t^{N-1} d t d \mathcal{H}^{N-1}(\sigma) \\
& =\int_{S^{N-1}} \Phi(|\nabla f(x) \cdot \sigma|) d \mu_{\varepsilon_{j}, \eta}(\sigma)
\end{aligned}
$$

Since $\Phi(|\nabla f(x) \cdot \sigma|)$ is continuous by Lemma 3.4 it follows

$$
\lim _{j \rightarrow+\infty} \int_{A^{\eta}} \Phi\left(\left|\nabla f(x) \frac{x-y}{|x-y|}\right|\right) \rho_{\varepsilon_{j}}^{\eta}(x-y) d \mathcal{L}^{N}(y)=\int_{S^{N-1}} \Phi(|\nabla f(x) \cdot \sigma|) d \mu(\sigma) .
$$


Lemma 3.9. Let $\Omega \subset \mathbb{R}^{N}$ be open, let $\Phi$ and $\rho_{\varepsilon}$ satisfy $H-1, H-2, H-3$, let $A \subset \Omega$ be open and bounded with dist $(A, \partial \Omega)>$ 0 , let $f \in W^{1} L^{\Phi}(\Omega)$; then for all $0<\eta<\frac{1}{3} \operatorname{dist}(A, \partial \Omega)$ we have

$$
\begin{aligned}
& \int_{A^{\eta}}\left(\int_{A^{\eta}} \Phi\left(\frac{|f(x)-f(y)|}{|x-y|}\right) \rho_{\varepsilon}^{\eta}(x-y) d \mathcal{L}^{N}(y)\right) d \mathcal{L}^{N}(x) \leq \\
& \leq \int_{A^{2 \eta}}\left(\int_{B_{\eta}(0)} \Phi\left(\left|\nabla f(x) \cdot \frac{h}{|h|}\right|\right) \rho_{\varepsilon}^{\eta}(h) d \mathcal{L}^{N}(h)\right) d \mathcal{L}^{N}(x) .
\end{aligned}
$$

Proof. Making the change of variables $y=x+h$, since $\rho_{\varepsilon_{j}}^{\eta}(y-x)=0$ if $|y-x|>\eta$ then

$$
\begin{aligned}
& \int_{A^{\eta}}\left(\int_{A^{\eta}} \Phi\left(\frac{|f(y)-f(x)|}{|y-x|}\right) \rho_{\varepsilon}^{\eta}(y-x) d \mathcal{L}^{N}(y)\right) d \mathcal{L}^{N}(x) \\
& =\int_{A^{\eta}}\left(\int_{B_{\eta}(x)} \Phi\left(\frac{|f(x+h)-f(x)|}{|h|}\right) \rho_{\varepsilon}^{\eta}(h) d \mathcal{L}^{N}(h)\right) d \mathcal{L}^{N}(x)
\end{aligned}
$$

For $0<\delta<\eta<\frac{\operatorname{dist}(A, \partial \Omega)}{3}$ the function $f_{\delta}$ is well defined in $A^{\eta}$, then we have

$$
\begin{aligned}
& \int\left(\int_{A^{\eta}} \Phi\left(\frac{\left|f_{\delta}(y)-f_{\delta}(x)\right|}{|y-x|}\right) \rho_{\varepsilon}^{\eta}(y-x) d \mathcal{L}^{N}(y)\right) d \mathcal{L}^{N}(x)= \\
& =\int_{A^{\eta}}\left(\int_{B_{\eta}(0)} \Phi\left(\frac{\left|f_{\delta}(x+h)-f_{\delta}(x)\right|}{|h|}\right) \rho_{\varepsilon}^{\eta}(h) d \mathcal{L}^{N}(h)\right) d \mathcal{L}^{N}(x) \\
& =\int_{A^{\eta}}\left(\int_{B_{\eta}(0)} \Phi\left(\int_{0}^{1}\left|\nabla f_{\delta}(x+t h) \cdot \frac{h}{|h|}\right| d t\right) \rho_{\varepsilon}^{\eta}(h) d \mathcal{L}^{N}(h)\right) d \mathcal{L}^{N}(x) \\
& =I
\end{aligned}
$$

Using Jensen's inequality we get

$$
I \leq \int_{A^{\eta}}\left(\int_{B_{\eta}(0)} \int_{0}^{1} \Phi\left(\left|\nabla f_{\delta}(x+t h) \cdot \frac{h}{|h|}\right|\right) d t \rho_{\varepsilon}^{\eta}(h) d \mathcal{L}^{N}(h)\right) d \mathcal{L}^{N}(x)
$$

Since $|h|<\eta$ by Tonelli's theorem it follows

$$
I \leq \int_{A^{2 \eta}}\left(\int_{B_{\eta}(0)} \Phi\left(\left|\nabla f_{\delta}(y) \cdot \frac{h}{|h|}\right|\right) \rho_{\varepsilon}^{\eta}(h) d \mathcal{L}^{N}(h)\right) d \mathcal{L}^{N}(y)
$$

and

$$
\begin{aligned}
& \int_{A^{\eta}}\left(\int_{A^{\eta}} \Phi\left(\frac{\left|f_{\delta}(y)-f_{\delta}(x)\right|}{|y-x|}\right) \rho_{\varepsilon}^{\eta}(y-x) d \mathcal{L}^{N}(y)\right) d \mathcal{L}^{N}(x) \leq \\
& \left.\leq \int_{A^{2 \eta}}\left(\int_{B_{\eta}(0)} \Phi\left(\mid \nabla f_{\delta}(y) \cdot \frac{h}{|h|}\right)\right) \rho_{\varepsilon}^{\eta}(h) d \mathcal{L}^{N}(h)\right) d \mathcal{L}^{N}(y)
\end{aligned}
$$

letting $\delta \rightarrow 0$ by Fatou's lemma and Lebesgue dominated convergence theorem we obtain (3.14).

Lemma 3.10. Let $\Omega \subset \mathbb{R}^{N}$ be open, let $\Phi$ and $\rho_{\varepsilon}$ satisfy $H-1, H-2, H-3$, let $A \subset \Omega$ be open and bounded with dist $(A, \partial \Omega)>$ 0 , let $f \in L^{\Phi}(\Omega)$; then for all $0<\delta<\eta<\frac{1}{3} \operatorname{dist}(A, \partial \Omega)$ we have

$$
\begin{aligned}
& \int_{A}\left(\int_{A} \Phi\left(\frac{\left|f_{\delta}(x)-f_{\delta}(y)\right|}{|x-y|}\right) \rho_{\varepsilon}^{\eta}(x-y) d \mathcal{L}^{N}(y)\right) d \mathcal{L}^{N}(x) \leq \\
& \int_{A^{\eta}}\left(\int_{A^{\eta}} \Phi\left(\frac{|f(x)-f(y)|}{|x-y|}\right) \rho_{\varepsilon}^{\eta}(x-y) d \mathcal{L}^{N}(y)\right) d \mathcal{L}^{N}(x)
\end{aligned}
$$

where $f_{\delta}$ is the mollification of $f$. 
Proof. Let us consider

$$
I_{0}=\int_{A}\left(\int_{A} \Phi\left(\frac{\left|f_{\delta}(x)-f_{\delta}(y)\right|}{|x-y|}\right) \rho_{\varepsilon}^{\eta}(x-y) d \mathcal{L}^{N}(y)\right) d \mathcal{L}^{N}(x)
$$

then, we get

$$
I_{0}=\int_{A}\left(\int_{A} \Phi\left(\frac{\left|\int_{B_{\delta}(0)} \psi_{\delta}(z) f(x-z)-f(y-z) d z\right|}{|x-y|}\right) \rho_{\varepsilon}^{\eta}(x-y) d \mathcal{L}^{N}(y)\right) d \mathcal{L}^{N}(x)
$$

and

$$
I_{0} \leq \int_{A}\left(\int_{A} \Phi\left(\int_{B_{\delta}(0)} \psi_{\delta}(z) \frac{|f(x-z)-f(y-z)|}{|x-y|} d z\right) \rho_{\varepsilon}^{\eta}(x-y) d \mathcal{L}^{N}(y)\right) d \mathcal{L}^{N}(x) .
$$

Since $\Phi$ is a convex function, using Remark 3.1, it follows

$$
I_{0} \leq \int_{A}\left(\int_{A}\left(\int_{B_{\delta}(0)} \psi_{\delta}(z) \Phi\left(\frac{|f(x-z)-f(y-z)|}{|x-y|}\right) d z\right) \rho_{\varepsilon}^{\eta}(x-y) d \mathcal{L}^{N}(y)\right) d \mathcal{L}^{N}(x)
$$

Using Tonelli's theorem we get

$$
I_{0} \leq \int_{B_{\delta}(0)} \int_{A}\left(\int_{A} \Phi\left(\frac{|f(x-z)-f(y-z)|}{|x-y|}\right) \rho_{\varepsilon}^{\eta}(x-y) d \mathcal{L}^{N}(y)\right) \psi_{\delta}(z) d \mathcal{L}^{N}(x) d z
$$

Then making the change of variables $w=x+z, u=y+z$, for $z \in B_{\delta}(0)$, since the integrand is non-negative, we have

$$
I_{0} \leq \int_{B_{\delta}(0)} \int_{A}\left(\int_{A} \Phi\left(\frac{|f(w)-f(u)|}{|w-u|}\right) \rho_{\varepsilon}^{\eta}(w-u) d \mathcal{L}^{N}(u)\right) d \mathcal{L}^{N}(w) \psi_{\delta}(z) d z
$$

Since $\int_{\mathbb{R}^{N}} \psi_{\delta}(z-y) d z=1$, it follows

$$
I_{0} \leq \int_{A}\left(\int_{A} \Phi\left(\frac{|f(w)-f(u)|}{|w-u|}\right) \rho_{\varepsilon}^{\eta}(w-u) d \mathcal{L}^{N}(u)\right) d \mathcal{L}^{N}(w)
$$

\section{Proof of Theorem 1.2}

Theorem 4.1. Let $\Omega \subset \mathbb{R}^{N}$ be open, let $\Phi$ and $\rho_{\varepsilon}$ satisfy $H-1, H-2, H-3$, let $\Phi \in \Delta_{2}$, let $f \in L_{\text {loc }}^{\Phi}(\Omega)$. Assume

$$
\lim _{\lambda \rightarrow 0} \limsup _{\varepsilon \rightarrow 0^{+}} \int_{\Omega_{\lambda}} \int_{\Omega_{\lambda}} \Phi\left(\frac{|f(x)-f(y)|}{|x-y|}\right) \rho_{\varepsilon}(x-y) d \mathcal{L}^{N}(y) d \mathcal{L}^{N}(x)<+\infty .
$$

Then $f \in W^{1} L_{\text {loc }}^{\Phi}(\Omega)$ and $\nabla f \in L^{\Phi}(\Omega)$. Moreover there exist $\varepsilon_{j} \rightarrow 0^{+}$and a probability measure $\mu$ in $\mathcal{M}\left(S^{N-1}\right)$ such that for every $0<\eta<\frac{\lambda}{3}$ then

$$
\begin{aligned}
& \lim _{\lambda \rightarrow 0} \liminf _{\varepsilon \rightarrow 0^{+}} \int_{\Omega_{\lambda}^{\eta}}\left(\int_{\Omega_{\lambda}^{2 \eta}} \Phi\left(\frac{|f(x)-f(y)|}{|x-y|}\right) \rho_{\varepsilon_{j}}(x-y) d \mathcal{L}^{N}(y)\right) d \mathcal{L}^{N}(x) \geq \\
& \left.\geq \iint_{\Omega} \int_{S^{N-1}} \Phi(|\nabla f(x) \cdot \sigma|) d \mu(\sigma)\right) d \mathcal{L}^{N}(x) .
\end{aligned}
$$


Proof. We define

$$
C=\lim _{\lambda \rightarrow 0} \limsup _{\varepsilon \rightarrow 0^{+}} \int_{\Omega_{\lambda}} \int_{\Omega_{\lambda}} \Phi\left(\frac{|f(x)-f(y)|}{|x-y|}\right) \rho_{\varepsilon}(x-y) d \mathcal{L}^{N}(y) d \mathcal{L}^{N}(x)<+\infty
$$

by the monotonicity of the integrals over $\Omega_{\lambda}$ we have that for any $\eta<\frac{\lambda}{3}$,

$$
\limsup _{\varepsilon \rightarrow 0^{+}} \int_{\Omega_{\lambda}^{\eta}} \int_{\Omega_{\lambda}^{2 \eta}} \Phi\left(\frac{|f(x)-f(y)|}{|x-y|}\right) \rho_{\varepsilon}(x-y) d \mathcal{L}^{N}(y) d \mathcal{L}^{N}(x) \leq C
$$

where $\Omega_{\lambda}^{\eta}=\left(\Omega_{\lambda}\right)^{\eta}$, since $\rho_{\varepsilon}^{\eta} \leq \rho_{\varepsilon}$, we get

$$
\limsup _{\varepsilon \rightarrow 0^{+}} \int_{\Omega_{\lambda}^{\eta}} \int_{\Omega_{\lambda}^{2 \eta}} \Phi\left(\frac{|f(x)-f(y)|}{|x-y|}\right) \rho_{\varepsilon}^{\eta}(x-y) d \mathcal{L}^{N}(y) d \mathcal{L}^{N}(x) \leq C
$$

Fix $0<\eta<\frac{\lambda}{3}$, for any $0<\delta<\eta$ apply Lemma 3.10 we obtain

$$
I_{\delta} \leq \int_{\Omega_{\lambda}^{\eta}} \int_{\Omega_{\lambda}^{2 \eta}} \Phi\left(\frac{|f(x)-f(y)|}{|x-y|}\right) \rho_{\varepsilon}^{\eta}(x-y) d \mathcal{L}^{N}(y) d \mathcal{L}^{N}(x)
$$

where

$$
I_{\delta}=\int_{\Omega_{\lambda}^{\eta}} \int_{\Omega_{\lambda}^{\eta}} \Phi\left(\frac{\left|f_{\delta}(x)-f_{\delta}(y)\right|}{|x-y|}\right) \rho_{\varepsilon}^{\eta}(x-y) d \mathcal{L}^{N}(y) d \mathcal{L}^{N}(x)
$$

Let $\mu_{\varepsilon}$ be the measures defined in (3.1). By Lemma 3.2 there exist a subsequence $\left\{\varepsilon_{j}\right\}$, with $\varepsilon_{j} \rightarrow 0$, and a probability measure $\mu \in \mathcal{M}\left(S^{N-1}\right)$ such that $\mu_{\varepsilon_{j}} \stackrel{*}{\rightarrow} \mu$ in $\mathcal{M}\left(S^{N-1}\right)$. Since $f_{\delta} \in C^{2}\left(\overline{\Omega_{\lambda}^{2 \eta}}\right)$ with $\Omega_{\lambda}^{2 \eta}$ open and bounded, by Lemma 3.8 , for every $x \in \Omega_{\lambda}$,

$$
\lim _{j \rightarrow+\infty} \int_{\Omega_{\lambda}^{\eta}} \Phi\left(\frac{\left|f_{\delta}(x)-f_{\delta}(y)\right|}{|x-y|}\right) \rho_{\varepsilon}^{\eta}(x-y) d \mathcal{L}^{N}(y)=\int_{S^{N-1}} \Phi\left(\left|\nabla f_{\delta}(x) \cdot \sigma\right|\right) d \mu(\sigma)
$$

Applying Fatou's lemma we have

$$
\begin{aligned}
& \left.\int_{\Omega_{\lambda}^{\eta}} \int_{S^{N-1}} \Phi\left(\left|\nabla f_{\delta}(x) \cdot \sigma\right|\right) d \mu(\sigma)\right) d \mathcal{L}^{N}(x) \leq \\
& \leq \liminf _{j \rightarrow+\infty} \int_{\Omega_{\lambda}^{\eta} \Omega_{\lambda}^{\eta}} \Phi\left(\frac{\left|f_{\delta}(x)-f_{\delta}(y)\right|}{|x-y|}\right) \rho_{\varepsilon_{j}}^{\eta}(x-y) d \mathcal{L}^{N}(y) d \mathcal{L}^{N}(x) \\
& \leq \int_{\Omega_{\lambda}^{\eta}} \int_{\Omega_{\lambda}^{2 \eta}} \Phi\left(\frac{|f(x)-f(y)|}{|x-y|}\right) \rho_{\varepsilon_{j}}^{\eta}(x-y) d \mathcal{L}^{N}(y) d \mathcal{L}^{N}(x) \leq C
\end{aligned}
$$

so that

$$
\int_{\Omega_{\lambda}}\left(\int_{S^{N-1}} \Phi\left(\left|\nabla f_{\delta}(x) \cdot \sigma\right|\right) d \mu(\sigma)\right) d \mathcal{L}^{N}(x) \leq C
$$

Then Lemma 3.2 implies

$$
\int_{\Omega_{\lambda}} \Phi\left(\left|\nabla f_{\delta}(x)\right|\right) d \mathcal{L}^{N}(x) \leq \frac{C}{\alpha}
$$

for some $\alpha>0$, independent of $\lambda$. Using the P-D theorem we get $\nabla f_{\delta} \rightarrow V$ in $L_{l o c}^{1}(\Omega)$. Since as $\delta \rightarrow 0, f_{\delta} \rightarrow f$ in $L_{l o c}^{1}(\Omega)$ then $f \in W^{1} L_{l o c}^{\Phi}(\Omega)$ and $\nabla f=V \in L^{\Phi}\left(\Omega_{\lambda}, \mathbb{R}^{N}\right)$. Finally, letting $\lambda \rightarrow 0$ we have $\nabla f \in L^{\Phi}\left(\Omega, \mathbb{R}^{N}\right)$. 
Theorem 4.2. Let $\Omega \subset \mathbb{R}^{N}$ be open, let $\Phi$ and $\rho_{\varepsilon}$ satisfy $H-1, H-2, H-3$, let $f \in W^{1} L_{\text {loc }}^{\Phi}(\Omega)$ and $\nabla f \in L^{\Phi}\left(\Omega\right.$, $\left.\mathbb{R}^{N}\right)$; then it follows

$$
\begin{aligned}
& \left.\iint_{\Omega_{\lambda}^{\eta}} \int_{\Omega_{\lambda}^{\eta}} \Phi\left(\frac{|f(x)-f(y)|}{|x-y|}\right) \rho_{\varepsilon}(x-y) d \mathcal{L}^{N}(y)\right) d \mathcal{L}^{N}(x) \leq \\
& \leq \int_{\Omega^{2}} \Phi(|\nabla f(x)|) d \mathcal{L}^{N}(x)+ \\
& +\frac{2^{m}}{\eta^{m}} \int_{\Omega_{\lambda}^{\eta}} \Phi(|f(x)|) d \mathcal{L}^{N}(x) \int_{|h|>\eta} \rho_{\varepsilon}(h) d \mathcal{L}^{N}(h)
\end{aligned}
$$

Proof. Fix $0<\lambda<1$ and $0<\eta<\frac{\lambda}{2}$; consider

$$
\begin{aligned}
& \int_{\Omega_{\lambda}^{\eta}}\left(\int_{\Omega_{\lambda}^{\eta}} \Phi\left(\frac{|f(x)-f(y)|}{|x-y|}\right) \rho_{\varepsilon}(x-y) d \mathcal{L}^{N}(y)\right) d \mathcal{L}^{N}(x)= \\
& \int_{\Omega_{\lambda}^{\eta}}\left(\int_{|x-y|<\eta} \Phi\left(\frac{|f(x)-f(y)|}{|x-y|}\right) \rho_{\varepsilon}(x-y) d \mathcal{L}^{N}(y)\right) d \mathcal{L}^{N}(x)+ \\
& \int_{\Omega_{\lambda}^{\eta}}\left(\int_{|x-y|>\eta} \Phi\left(\frac{|f(x)-f(y)|}{|x-y|}\right) \rho_{\varepsilon}(x-y) d \mathcal{L}^{N}(y)\right) d \mathcal{L}^{N}(x)
\end{aligned}
$$

Considering

$$
\begin{aligned}
B & =\int_{\Omega_{\lambda}^{\eta}}\left(\int_{x-y \mid>\eta} \Phi\left(\frac{|f(x)-f(y)|}{|x-y|}\right) \rho_{\varepsilon}(x-y) d \mathcal{L}^{N}(y)\right) d \mathcal{L}^{N}(x) \\
& \leq \frac{2^{m-1}}{\eta^{m}} \int_{\Omega_{\lambda}^{\eta}}\left(\int_{\langle x-y|>\eta}(\Phi(|f(x)|)+\Phi(|f(y)|)) \rho_{\varepsilon}(x-y) d \mathcal{L}^{N}(y)\right) d \mathcal{L}^{N}(x)
\end{aligned}
$$

By Fubini and Tonelli Theorems we get

$$
\begin{aligned}
B \leq & \frac{2^{m-1}}{\eta^{m}} \int_{\Omega_{\lambda}^{\eta}} \Phi(|f(x)|)\left(\int_{x-y \mid>\eta} \rho_{\varepsilon}(x-y) d \mathcal{L}^{N}(y)\right) d \mathcal{L}^{N}(x)+ \\
& +\frac{2^{m-1}}{\eta^{m}} \int_{\Omega_{\lambda}^{\eta}} \Phi(|f(y)|)\left(\int_{x-y \mid>\eta} \rho_{\varepsilon}(x-y) d \mathcal{L}^{N}(x)\right) d \mathcal{L}^{N}(y)
\end{aligned}
$$

and

$$
B \leq \frac{2^{m}}{\eta^{m}} \int_{\Omega_{\lambda}^{\eta}} \Phi(|f(x)|) d \mathcal{L}^{N}(x) \int_{|h|>\eta} \rho_{\varepsilon}(h) d \mathcal{L}^{N}(h)
$$

Moreover applaying Lemma 3.9 we get

$$
\begin{aligned}
& \left.\int_{\Omega_{\lambda}^{\eta}} \int_{|x-y|<\eta} \Phi\left(\frac{|f(x)-f(y)|}{|x-y|}\right) \rho_{\varepsilon}(x-y) d \mathcal{L}^{N}(y)\right) d \mathcal{L}^{N}(x) \\
& \leq \int_{\Omega_{\lambda}^{\eta}}\left(\int_{x-y \mid<\eta} \Phi\left(\frac{|f(x)-f(y)|}{|x-y|}\right) \rho_{\varepsilon}^{\eta}(x-y) d \mathcal{L}^{N}(y)\right) d \mathcal{L}^{N}(x) \\
& \leq \int_{\Omega_{\lambda}^{\eta}}\left(\int_{B_{\eta}(0)} \Phi\left(\left|\nabla f(x) \cdot \frac{h}{|h|}\right|\right) \rho_{\varepsilon}^{\eta}(h) d \mathcal{L}^{N}(h)\right) d \mathcal{L}^{N}(x) \\
& \leq \int_{\Omega} \Phi(|\nabla f(x)|) d \mathcal{L}^{N}(x)
\end{aligned}
$$

then we get (4.3). 
Now we can show the Theorem 1.2

Proof. (Proof of Theorem 1.2) Let $f \in L_{l o c}^{\Phi}(\Omega)$, assume

$$
\lim _{\lambda \rightarrow 0} \limsup _{\varepsilon \rightarrow 0^{+}} \int_{\Omega_{\lambda}} \int_{\Omega_{\lambda}} \Phi\left(\frac{|f(x)-f(y)|}{|x-y|}\right) \rho_{\varepsilon}(x-y) d \mathcal{L}^{N}(y) d \mathcal{L}^{N}(x)<+\infty
$$

then applaying Theorem 4.1 we have $f \in W^{1} L_{l o c}^{\Phi}(\Omega)$ and $\nabla f \in L^{\Phi}\left(\Omega, \mathbb{R}^{N}\right)$. Moreover we get

$$
\begin{aligned}
& \liminf _{j \rightarrow+\infty} \int_{\Omega_{\lambda}^{\eta}}\left(\int_{\Omega_{\lambda}^{2 \eta}} \Phi\left(\frac{|f(x)-f(y)|}{|x-y|}\right) \rho_{\varepsilon_{j}}(x-y) d \mathcal{L}^{N}(y)\right) d \mathcal{L}^{N}(x) \geq \\
& \geq \int_{\Omega}\left(\int_{S^{N-1}} \Phi(|\nabla f(x) \cdot \sigma|) d \mu(\sigma)\right) d \mathcal{L}^{N}(x) .
\end{aligned}
$$

Conversely let $f \in W^{1} L_{l o c}^{\Phi}(\Omega)$ and $\nabla f \in L^{\Phi}\left(\Omega, \mathbb{R}^{N}\right)$; then using Theorem 4.2 we have

$$
\begin{aligned}
& \int_{\Omega_{\lambda}^{\eta}}\left(\int_{\Omega_{\lambda}^{\eta}} \Phi\left(\frac{|f(x)-f(y)|}{|x-y|}\right) \rho_{\varepsilon}(x-y) d \mathcal{L}^{N}(y)\right) d \mathcal{L}^{N}(x) \leq \\
& \leq \int_{\Omega} \Phi(|\nabla f(x)|) d \mathcal{L}^{N}(x)+ \\
& +\frac{2^{m}}{\eta^{m}} \int_{\Omega_{\lambda}^{\eta}} \Phi(|f(x)|) d \mathcal{L}^{N}(x) \int_{|h|>\eta} \rho_{\varepsilon}(h) d \mathcal{L}^{N}(h)
\end{aligned}
$$

Since, by Remark 3.5, $\lim _{\varepsilon \rightarrow 0^{+}} \int_{|h|>\eta} \rho_{\varepsilon}(h) d \mathcal{L}^{N}(h)=0$ we get

$$
\limsup _{\varepsilon \rightarrow 0^{+}} \int_{\Omega_{\lambda}^{\eta}}\left(\int_{\Omega_{\lambda}^{\eta}} \Phi\left(\frac{|f(x)-f(y)|}{|x-y|}\right) \rho_{\varepsilon}(x-y) d \mathcal{L}^{N}(y)\right) d \mathcal{L}^{N}(x) \leq \int_{\Omega} \Phi(|\nabla f(x)|) d \mathcal{L}^{N}(x)
$$

and

$$
\lim _{\lambda \rightarrow 0^{+}} \limsup _{\varepsilon \rightarrow 0^{+}} \int_{\Omega_{\lambda}^{\eta}}\left(\int_{\Omega_{\lambda}^{\eta}} \Phi\left(\frac{|f(x)-f(y)|}{|x-y|}\right) \rho_{\varepsilon}(x-y) d \mathcal{L}^{N}(y)\right) d \mathcal{L}^{N}(x)<+\infty
$$

Moreover, since, by Lemma 3.9,

$$
\begin{aligned}
& \left.\int_{\Omega_{\lambda}^{\eta}} \int_{|x-y|<\eta} \int_{\Omega_{\lambda}^{\eta}} \Phi\left(\frac{|f(x)-f(y)|}{|x-y|}\right) \rho_{\varepsilon}(x-y) d \mathcal{L}^{N}(y)\right) d \mathcal{L}^{N}(x) \\
& \left.\leq \int_{|x-y|<\eta} \Phi\left(\frac{|f(x)-f(y)|}{|x-y|}\right) \rho_{\varepsilon}^{\eta}(x-y) d \mathcal{L}^{N}(y)\right) d \mathcal{L}^{N}(x) \\
& \leq \int_{\Omega_{\lambda}^{\eta}}\left(\int_{B_{\eta}(0)} \Phi\left(\left|\nabla f(x) \cdot \frac{h}{|h|}\right|\right) \rho_{\varepsilon}^{\eta}(h) d \mathcal{L}^{N}(h)\right) d \mathcal{L}^{N}(x)
\end{aligned}
$$

we have

$$
\begin{aligned}
& \liminf _{j \rightarrow+\infty} \int_{\Omega_{\lambda}^{\eta}}\left(\int_{|x-y|<\eta} \Phi\left(\frac{|f(x)-f(y)|}{|x-y|}\right) \rho_{\varepsilon_{j}}(x-y) d \mathcal{L}^{N}(y)\right) d \mathcal{L}^{N}(x) \\
& \left.\leq \liminf _{j \rightarrow+\infty} \int_{\Omega_{\lambda}^{2 \eta}} \int_{B_{\eta}(0)} \Phi\left(\left|\nabla f(x) \cdot \frac{h}{|h|}\right|\right) \rho_{\varepsilon_{j}}^{\eta}(h) d \mathcal{L}^{N}(h)\right) d \mathcal{L}^{N}(x) \\
& \left.=\liminf _{j \rightarrow+\infty} \int_{\Omega_{\lambda}^{2 \eta}} \int_{S^{N-1}} \Phi(|\nabla f(x) \cdot \sigma|) d \mu_{\varepsilon_{j}}^{\eta}(\sigma)\right) d \mathcal{L}^{N}(x) \\
& \left.=\int_{\Omega_{\lambda}^{2 \eta}} \int_{S^{N-1}} \Phi(|\nabla f(x) \cdot \sigma|) d \mu(\sigma)\right) d \mathcal{L}^{N}(x)
\end{aligned}
$$


Conbining (4.4) and (4.5) we get

$$
\begin{aligned}
& \int_{\Omega_{\lambda}} \int_{S^{N-1}} \int_{j(|\nabla f(x) \cdot \sigma|) d \mu(\sigma)) d \mathcal{L}^{N}(x)} \\
\leq & \liminf _{j \rightarrow+\infty} \int_{\Omega_{\lambda}^{\eta}}\left(\int_{x-y \mid<\eta} \Phi\left(\frac{|f(x)-f(y)|}{|x-y|}\right) \rho_{\varepsilon_{j}}(x-y) d \mathcal{L}^{N}(y)\right) d \mathcal{L}^{N}(x) \\
\leq & \limsup _{j \rightarrow+\infty} \int_{\Omega_{\lambda}^{\eta}}\left(\int_{x-y \mid<\eta} \Phi\left(\frac{|f(x)-f(y)|}{|x-y|}\right) \rho_{\varepsilon_{j}}(x-y) d \mathcal{L}^{N}(y)\right) d \mathcal{L}^{N}(x) \\
\leq & \left.\int_{\Omega} \int_{S^{N-1}} \Phi(|\nabla f(x) \cdot \sigma|) d \mu(\sigma)\right) d \mathcal{L}^{N}(x)
\end{aligned}
$$

sending $\lambda \rightarrow 0$ it follows

$$
\begin{aligned}
& \lim _{\lambda \rightarrow 0^{+}} \lim _{\varepsilon \rightarrow 0^{+}} \iint_{\Omega_{\lambda}}\left(\int_{\Omega_{\lambda}} \Phi\left(\frac{|f(x)-f(y)|}{|x-y|}\right) \rho_{\varepsilon}(x-y) d \mathcal{L}^{N}(y)\right) d \mathcal{L}^{N}(x)= \\
& \left.=\iint_{\Omega} \int_{S^{N-1}} \Phi(|\nabla f(x) \cdot \sigma|) d \mu(\sigma)\right) d \mathcal{L}^{N}(x)
\end{aligned}
$$

When $\rho_{\varepsilon}$ satisfy H-4 we get $\mu=\mathcal{H}^{N-1}$ then

$$
\int_{S^{N-1}} \Phi(|\nabla f(x) \cdot \sigma|) d \mu(\sigma)=\int_{S^{N-1}} \Phi\left(|\nabla f(x)|\left|\frac{\nabla f(x)}{|\nabla f(x)|} \cdot \sigma\right|\right) d \mathcal{H}^{N-1}(\sigma)
$$

Since $\frac{\nabla f(x)}{|\nabla f(x)|} \in S^{N-1}$ using the rotational invariance of $\mathcal{H}^{N-1}$ then

$$
\int_{S^{N-1}} \Phi(|\nabla f(x) \cdot \sigma|) d \mu(\sigma)=\int_{S^{N-1}} \Phi\left(|\nabla f(x)|\left|e_{1} \cdot \sigma\right|\right) d \mathcal{H}^{N-1}(\sigma)
$$

Since $\left|e_{1} \cdot \sigma\right| \leq 1$, by Remark 2.18 , we get

$$
\int_{S^{N-1}} \Phi\left(|\nabla f(x)|\left|e_{1} \cdot \sigma\right|\right) d \mathcal{H}^{N-1}(\sigma) \leq \Phi(|\nabla f(x)|) \int_{S^{N-1}}\left|e_{1} \cdot \sigma\right| d \mathcal{H}^{N-1}(\sigma)
$$

Moredover by Jensen inequality we have

$$
\frac{1}{\mathcal{H}^{N-1}\left(S^{N-1}\right)} \int_{S^{N-1}} \Phi\left(|\nabla f(x)|\left|e_{1} \cdot \sigma\right|\right) d \mathcal{H}^{N-1}(\sigma) \geq \Phi\left(\frac{|\nabla f(x)|}{\mathcal{H}^{N-1}\left(S^{N-1}\right)} \int_{S^{N-1}}\left|e_{1} \cdot \sigma\right| d \mathcal{H}^{N-1}(\sigma)\right)
$$

If we suppose $\Phi \in$ on $(0,+\infty)$, since $\frac{1}{\mathcal{H}^{N-1}\left(S^{N-1}\right)} \int_{S^{N-1}}\left|e_{1} \cdot \sigma\right| d \mathcal{H}^{N-1}(\sigma) \leq \frac{1}{\mathcal{H}^{N-1}\left(S^{N-1}\right)} \int_{S^{N-1}}\left|e_{1}\right||\sigma| d \mathcal{H}^{N-1}(\sigma) \leq 1$, using Proposition 2.21 it follows

$$
\frac{1}{\mathcal{H}^{N-1}\left(S^{N-1}\right)} \int_{S^{N-1}} \Phi\left(|\nabla f(x)|\left|e_{1} \cdot \sigma\right|\right) d \mathcal{H}^{N-1}(\sigma) \geq \Phi(|\nabla f(x)|)\left(\Lambda_{N}\right)^{m}
$$

where $\Lambda_{N}=\frac{1}{\mathcal{H}^{N-1}\left(S^{N-1}\right)} \int_{S^{N-1}}\left|e_{1} \cdot \sigma\right| d \mathcal{H}^{N-1}(\sigma)$, then

$$
\int_{S^{N-1}} \Phi\left(|\nabla f(x)|\left|e_{1} \cdot \sigma\right|\right) d \mathcal{H}^{N-1}(\sigma) \geq w_{m, N} \cdot \Phi(|\nabla f(x)|) \int_{S^{N-1}}\left|e_{1} \cdot \sigma\right| d \mathcal{H}^{N-1}(\sigma)
$$


where $w_{m, N}=\left(\frac{1}{\mathcal{H}^{N-1}\left(S^{N-1}\right)} \int_{S^{N-1}}\left|e_{1} \cdot \sigma\right| d \mathcal{H}^{N-1}(\sigma)\right)^{m-1}>0$. Using (4.8) and (4.9) it follows

$$
w_{m} \leq \frac{\int_{\Omega}\left(\int_{S^{N-1}} \Phi(|\nabla f(x) \cdot \sigma|) d \mu(\sigma)\right) d \mathcal{L}^{N}(x)}{\int_{S^{N-1}}\left|e_{1} \cdot \sigma\right| d \mathcal{H}^{N-1}(\sigma) \int_{\Omega} \Phi(|\nabla f(x)|) d \mathcal{L}^{N}(x)} \leq 1
$$

then there exits $\Gamma_{m, N} \in\left[w_{m, N}, 1\right]$ such that

$$
\int_{\Omega}\left(\int_{S^{N-1}} \Phi(|\nabla f(x) \cdot \sigma|) d \mu(\sigma)\right) d \mathcal{L}^{N}(x)=\Gamma_{m_{, N}} \int_{S^{N-1}}\left|e_{1} \cdot \sigma\right| d \mathcal{H}^{N-1}(\sigma) \int_{\Omega} \Phi(|\nabla f(x)|) d \mathcal{L}^{N}(x)
$$

(1.8) follows if we define $k_{m, N}=\Gamma_{m_{, N}} \int_{S^{N-1}}\left|e_{1} \cdot \sigma\right| d \mathcal{H}^{N-1}(\sigma)$.

The proof of the Theorem 1.3 follows the ideas introduced in [17], we introduce it only for completeness.

Proof. (Proof of Theorem 1.3) Let $f \in L^{\Phi}(\Omega)$ satisfy (1.9) then for every $\eta \in\left(0, \frac{\lambda}{3}\right)$ and every $\Omega_{\lambda} \subset \Omega$ we get

$$
\limsup _{\epsilon \rightarrow 0} \int_{\Omega_{\lambda}^{\eta}} \int_{\Omega_{\lambda}^{2 \eta}} \Phi\left(\frac{|f(x)-f(y)|}{d_{\Omega}(x, y)}\right) \rho_{\epsilon}^{\eta}\left(d_{\Omega}(x, y)\right) d \mathcal{L}^{N}(y) d \mathcal{L}^{N}(x)<+\infty
$$

Since $\rho_{\epsilon}^{\eta}\left(d_{\Omega}(x, y)\right)=0$ if $d_{\Omega}(x, y)>\eta$ and $|x-y| \leq d_{\Omega}(x, y) \leq \eta<\frac{\lambda}{3}$ then for $x \in \Omega_{\lambda}^{\eta}$ and $y \in \Omega_{\lambda}^{2 \eta}$ the segment containing $x$ and $y$ is conteined in $\Omega$, it follows that $d_{\Omega}(x, y)=|x-y|$ and

$$
\limsup _{\epsilon \rightarrow 0} \int_{\Omega_{\lambda}^{\eta} \int_{\lambda}^{2 \eta}} \Phi\left(\frac{|f(x)-f(y)|}{|x-y|}\right) \rho_{\epsilon}^{\eta}(|x-y|) d \mathcal{L}^{N}(y) d \mathcal{L}^{N}(x)<+\infty
$$

then by Theorem $1.2 f \in W^{1, \Phi}(\Omega)$.

Now we observe as the hypothesis H-1 is technical and as we are able weakened.

Remark 4.3. If $\Phi \in \Delta_{2}^{m}$ on $\left(t_{0},+\infty\right)$ with $t_{0}>0$ then there exists $\Phi_{1} \in \Delta_{2}^{m}$ on $(0,+\infty)$ such that $\Phi \sim \Phi_{1}$. Moreover there exist $c_{1}, c_{2}, c_{3}, c_{4} \in \mathbb{R}^{+}$such that

$$
\Phi(t) \leq c_{1} \Phi_{1}(t)+c_{2}
$$

for every $t>0$ and

$$
\Phi_{1}(t) \leq c_{3} \Phi(t)+c_{4}
$$

for every $t>0$. Let us consider

$$
\int_{\Omega_{\lambda}} \int_{\Omega_{\lambda}} \Phi\left(\frac{|f(x)-f(y)|}{|x-y|}\right) \rho_{\varepsilon}(x-y) d \mathcal{L}^{N}(y) d \mathcal{L}^{N}(x)
$$

and

$$
\int_{\Omega_{\lambda}} \int_{\Omega_{\lambda}} \Phi_{1}\left(\frac{|f(x)-f(y)|}{|x-y|}\right) \rho_{\varepsilon}(x-y) d \mathcal{L}^{N}(y) d \mathcal{L}^{N}(x)
$$

using (4.10) and (4.11) we get

$$
\begin{aligned}
\iint_{\Omega_{\lambda}} \Phi\left(\frac{|f(x)-f(y)|}{|x-y|}\right) \rho_{\varepsilon}(x-y) d \mathcal{L}^{N}(y) d \mathcal{L}^{N}(x) & \leq c_{1} \iint_{\Omega_{\lambda}} \Phi_{\Omega_{\lambda}} \Phi_{1}\left(\frac{|f(x)-f(y)|}{|x-y|}\right) \rho_{\varepsilon}(x-y) d \mathcal{L}^{N}(y) d \mathcal{L}^{N}(x) \\
& +c_{2} \int_{\Omega_{\lambda}} \int_{\Omega_{\lambda}} \rho_{\varepsilon}(x-y) d \mathcal{L}^{N}(y) d \mathcal{L}^{N}(x)
\end{aligned}
$$


and

$$
\begin{aligned}
\iint_{\Omega_{l}} \int_{\Omega_{\lambda}} \Phi_{1}\left(\frac{|f(x)-f(y)|}{|x-y|}\right) \rho_{\varepsilon}(x-y) d \mathcal{L}^{N}(y) d \mathcal{L}^{N}(x) & \leq c_{3} \iint_{\Omega_{\lambda}} \int_{\Omega_{\lambda}} \Phi\left(\frac{|f(x)-f(y)|}{|x-y|}\right) \rho_{\varepsilon}(x-y) d \mathcal{L}^{N}(y) d \mathcal{L}^{N}(x) \\
& +c_{4} \int_{\Omega_{\lambda}} \int_{\Omega_{\lambda}} \rho_{\varepsilon}(x-y) d \mathcal{L}^{N}(y) d \mathcal{L}^{N}(x)
\end{aligned}
$$

Since $W^{1} L^{\Phi}(\Omega)=W^{1} L^{\Phi_{1}}(\Omega)$, see (Adams, 1975; Krasnosel'skij E Rutickii, 1961; Rao E Ren, 1991), then, by (4.12) and (4.13), Theorem 1.2 holds olso if $\Phi$ is a $N$-function and $\Phi \in \Delta_{2}$ on $\left(t_{0},+\infty\right)$ with $t_{0}>0$.

\section{References}

Adams, R. (1975). Sobolev spaces, Academic Press, New York.

Astarita, G., \& Marrucci, G. (1974). Principles of Non-Newtonian Fluid Mechanics, McGraw-Hill, London.

Bourgain, J., Brezis, H., \& Mironescu, P. (2001). Another look at Sobolev spaces. In J. L. Menaldi et al.. (eds.) Optimal control and partial diferential equations, 439-455, IOS Press.

Bourgain, J., Brezis, H., \& Mironescu, P. Limiting embedding theorems for $W^{s, p}$ when $s \rightarrow 1^{-}$and applications, J. Funct. Anal., 87, 77-101.

Breit, D., Stroffolini, B., \& Verde, A. (2011). A general regularity theorem for functionals with $\varphi$-growth, J. Math. Anal. Appl., 383, 226-233. https://doi.org/10.1016/j.jmaa.2011.05.012

Cianchi, A., \& Fusco, N. (1999). Gradient regularity for minimizers under general growth conditions, J. reine angew. Math., 507, 15-36. https://doi.org/10.1515/crll.1999.507.15

Dall'Aglio, A., Mascolo, E., \& Papi, G. (1998). Regularity for local minima of functionala with nonstandard growth conditions, Rend. Mat., 18(VII), 305-326.

Di Castro, A., Kuusi, T., \& Palatucci, G. (2016). Local behavior of fractional p-minimizers, Ann. Inst. H. Poincar Anal. Nonlin., 33, 1279-1299.

Di Castro, A., Kuusi, T., \& Palatucci, G. (2014). Nonlocal Harnack Inequalities, J. Funct. Anal., 267(6), 1897-1836. https://doi.org/10.1016/j.jfa.2014.05.023

Diening, L., \& Ruzika, M. (2007). Non-Newtonian fluids and function spaces, Nonlinear Analysis, Function Spaces and Applications, 8, 95-143

Diening, L., Stroffolini, B., \& Verde, A. (2009). Everywhere regulaity of functional with $\varphi$-growth. Manus. Math., 129, 440-481. https://doi.org/10.1007/s00229-009-0277-0

Fuchs, M. (2011). Local Lipschitz regularity of vector valued local minimizers of variational integrals with densities depending on the modulus of the gradient, Math. Nachrichten, 284, 266-272.

Fusco, N. \& Sbordone, C. (1990). Higher integrability of the gradient of minimizers of functionals with nonstandard growth conditions, Comm. pure appl. Math., XLIII, 673-683.

Gagliardo, E. (1957). Caratterizazioni delle tracce sulla frontiera relative ad alcune classi di funzioni in $\mathrm{n}$ variabili, Rend. Sem. Mat. Univ. Padova, 27, 284-305

Gosez, J. P. (1974). Nonlinear elliptic problems for equations with rapidly (or slowly) increasing coefficents, Trans. Amer. Math. Soc., 190, 163-205.

Granucci, T. (2017). An Harnack inequality for the quasi-minima of scalar integral functionals with general growth conditions, manuscripta math., 152, 345-380. https/doi.org/10.1007/s00229-016-0861-z

Leoni, G. \& Spector, D. (2011) Characterization of Sobolev and BV Spaces, J. Funct. Anal., 261, 2926-2958.

Lieberman, G. M. (1991). The Natural Generalization of the Natural Conditions of Ladyzhenskaya and Ural'tseva for Elliptic Equations, Comm. Part. Diff. Equat., 16, 331-361.

Klimov, V. S. (2000). Embedding Theorems and Continuity of Generalized Solutions of Quasilinear Elliptic Equations, Diff. Equations, 36, 6, 870-877, translated from Diff. Uravn., 784-791.

Krasnosel'skij, M. A., \& Rutickii, Ya. B. (1961). Convex Function and Orlicz Spaces, Noordhoff, Groningen.

Maz'ya, V., \& Shaposhnikova, T. (2002). On Bourgain, Brezis and Mironescu theorem concerning limiting embeddings of fractional Sobolev Spaces. J. Funct. Anal., 195, 230-238 
Mengesha, T., \& Spector, D. Localization of nonlocal gradients in various topologies. http/www.cvgmt.sns.it/paper/2211

Milman, M. (2005). Notes on limits of Sobolev spaces and the continuity of interpolation scales, Trans. Amer. Mat. Soc., $357,3425-3442$.

Ponce, A. (2004). A new approach to Sobolev space and connections to $\Gamma$-convergence. Calc. Var. and PDEs, 19, 229-255. https://doi.org/10.1007/s00526-003-0195-z

Rao, M. M., \& Ren, Z. D. (1991). Theory of Orlicz spaces, Marcel Dekker, New York.

Schikarra, A., Shieh, T. T., \& Spector, D. Regularity for a fractional p-Laplace equation. http/www.cvgmt.sns.it/paper/3145

Shieh, T. T., \& Spector, D. On a new class of fractional partial differential equations. http/www.cvgmt.sns.it/paper/2292

Talenti, G. (1990). Bounddeness of minimizers. Hokkaido Math. J., 19, 259-279. https://doi.org/10.14492/hokmj/1381517360

Young, W. H. (1912). On a classes of summable functions and their Fourier series. Proc. Royal Soc., Series (A), 87, 225-229. https://doi.org/10.1098/rspa.1912.0076

\section{Copyrights}

Copyright for this article is retained by the author(s), with first publication rights granted to the journal.

This is an open-access article distributed under the terms and conditions of the Creative Commons Attribution license (http://creativecommons.org/licenses/by/4.0/). 